\title{
miR-145-5p: A Potential Biomarker in Predicting Gleason Upgrading of Prostate Biopsy Samples Scored $3+3=6$
}

\author{
Tao Wang ${ }^{1, *}$ \\ Lei Dong ${ }^{2, *}$ \\ Juanjuan $\operatorname{Sun}^{3, *}$ \\ Jialiang Shao' \\ Jian Zhang' \\ Siteng Chen' \\ Chaofu Wang $\mathbb{D}^{2}$ \\ Gangfeng $\mathrm{Wu}^{4}$ \\ Xiang Wang'
}

'Department of Urology, Shanghai General Hospital, Shanghai Jiao Tong University School of Medicine, Shanghai, People's Republic of China; ${ }^{2}$ Department of Pathology, Ruijin Hospital, Shanghai Jiao Tong University School of Medicine, Shanghai, People's Republic of China; ${ }^{3}$ Department of Pathology, Shanghai General Hospital, Shanghai Jiao Tong University School of Medicine, Shanghai, People's Republic of China; ${ }^{4}$ Department of Urology, Shaoxing People's Hospital, Shaoxing, Zhejiang, People's Republic of China

*These authors contributed equally to this work

Correspondence: Gangfeng Wu

Department of Urology, Shaoxing People's Hospital, No. 568 Zhongxing North Road, Shaoxing, Zhejiang, 312000, People's Republic of China

Email gaffwu@sina.com

Xiang Wang

Department of Urology, Shanghai General Hospital, Shanghai Jiao Tong University

School of Medicine, Shanghai, 200080,

People's Republic of China

Email xiang.wangl@shgh.cn
Background: The Gleason grading system is a major tool used for prediction of prostate cancer (PCa) behavior. Because of heterogeneity and sampling errors, prognosis is variable even among patients with the same Gleason score (GS). Therefore, more accurate biomarkers that complement the Gleason system are needed to improve the clinical management of PCa. Methods: Formalin-fixed, paraffin embedded tissue samples were obtained from radical prostatectomy (RP) (patient set $1, n=53$ ) and needle biopsy (patient set 2, $n=107$; patient set $3, n=119$ ). Cancer tissues from pure regions of each Gleason pattern (GP) were separately collected using laser-captured microdissection, followed by Real-time-PCR to determine the relative expression of miRNAs, including miR-1-5p, miR-21-5p, miR-30d-5p, miR-100-5p, miR-145-5p, miR-224-5p, and miR-708-5p. miRNA's association with Gleason upgrading (GU) was evaluated using receiver operator characteristics (ROC) curve and multivariate logistic regression analysis. The integrated miRNA targets prediction and enrichment analyses were performed to determine the potential functions of miRNA.

Results: It was found that miR-145-5p in GP3 from radical prostatectomy (RP) were overexpressed in patients with GS6 PCa compared with GS7 patients, which was further confirmed in a larger biopsy cohort. ROC curve analysis revealed that miR-145-5p in biopsy was significantly associated with GU upon RP. In multivariate analyses, miR-145-5p was an independent predictor of GU.

Conclusion: Our study indicated that differential expression of miRNAs existed in GP3 from pure GS6 and GS7 PCa, highlighting a path toward the clinical use of miRNAs in predicting GU and assisting in treatment modality selection.

Keywords: prostate cancer, microRNA, biopsy, active surveillance, Gleason upgrading

\section{Introduction}

Prostate cancer $(\mathrm{PCa})$ is the most prevalent form of male malignant tumor in developed countries. There is a wide geographic variation in PCa incidence, and mortality rates differ among regions. ${ }^{1}$ In China, $\mathrm{PCa}$ is the second most common malignant tumor type observed in the male urogenital system after bladder carcinoma. ${ }^{2}$ The Gleason grading system, based on the architectural pattern of malignant glands, is a major tool used for prediction of $\mathrm{PCa}$ behavior, providing guidance for the treatment option. ${ }^{3,4}$ Because of PCa heterogeneity and sampling errors, prognosis is variable, even among patients with the same Gleason score (GS). ${ }^{5}$ Therefore, identification of more objective and accurate biomarkers that complement the Gleason grading system could improve the clinical management of PCa. 
MicroRNAs (miRNAs) are a class of small non-protein -coding RNAs that induce mRNA degradation or inhibit protein translation via direct interaction with complementary recognition sites of target mRNAs. ${ }^{6}$ The miRNA system regulates various physiological processes, including cellular proliferation, differentiation and apoptosis. ${ }^{7}$ Increasing evidence suggests that dysregulation of individual miRNAs or altered expression profiles of subsets of miRNAs in PCa could promote tumor progression, invasion, and metastasis. ${ }^{8-10}$ Besides, the evaluation of miRNA has advantages compared with that of mRNA, as miRNAs are stable and can be efficiently extracted from formalin-fixed tissues.

In this context, dysregulated miRNAs represent potentially useful biomarkers for $\mathrm{PCa}$ diagnosis, prognosis, and drug resistance. ${ }^{11-14}$ For example, miR-375 was reported to be significantly associated with lymph node involvement and metastases of $\mathrm{PCa}^{12}$ Serum miRNAs in the miR-200 and miR-17 families were associated with a prostate-specific antigen (PSA) response and improved overall survival in castrate-resistant prostate cancer receiving treatment with docetaxel. ${ }^{12}$ By combining bioinformatics and experimental analysis, a recent study showed that miR-210-3p, miR-23c, miR-592, and miR-93-5p were suitable biomarkers for $\mathrm{PCa}$ aggressiveness with regards to different GS. ${ }^{15}$ Furthermore, $\mathrm{PCa}$ in younger patients was recently found to have a different miRNA expression profile compared to that occurring in older man. ${ }^{16}$

Herein, the purpose of this study is to investigate the expression levels of seven miRNAs shown in the literature to be related to PCa: miR-1-5p, ${ }^{17,18} \mathrm{miR}-21-5 \mathrm{p},{ }^{19-21} \mathrm{miR}-$ 30d-5p, ${ }^{22,23}$ miR-100-5p, ${ }^{24,25}$ miR-145-5p, ${ }^{20,26,27}$ miR$224-5 \mathrm{p},{ }^{28-30}$ and miR-708-5p. ${ }^{25,31}$ We analyzed the expression of these miRNAs in pure regions of sections from each Gleason pattern (GP) and compared their expression with GS.

\section{Materials and Methods Human Tissue Samples}

A total of 53 formalin-fixed, paraffin-embedded (FFPE) radical prostatectomy (RP) samples and 107 FFPE needle biopsy $\mathrm{PCa}$ samples were obtained from the Fudan University Shanghai Cancer Center between January 2008 and April 2015 (patient set 1 and 2). Another 119 FFPE needle biopsy PCa samples were obtained from the Shanghai General Hospital between December 2012 and March 2018. Patient profiles are summarized as follows: patient set 1 (RP samples), $n=53$, including GP3: n=29, GP4: n=44, GP5: n=16, and adjacent normal tissue: $\mathrm{n}=53$ (Table 1); patient set 2 (biopsy samples), $\mathrm{n}=107$, including GP3 from the real GS6 cancer tissues, $n=32$, as were all confirmed in the following RP, and GP3 from GS7 in the biopsy tissues, $\mathrm{n}=75$ (Table 1); patient set 3 (biopsy samples), $n=119$, all were GS6 in the biopsy, including 66 samples with the real GS6 and 53 samples with GS $\geq 7$ in the subsequent RP (Table 2). Written informed consent was obtained from each patient included and the protocol of all study cohorts were approved by the Clinical Research Ethics Committee of Shanghai general hospital and the Ethics Committee of Fudan University Shanghai Cancer Center. All methods were performed in accordance with the Declaration of Helsinki. The 2014 ISUP modified Gleason scoring system was used to explore the Gleason scoring in PCa samples. ${ }^{4}$ All slides were reviewed by two board certified pathologists for the identification of each Gleason grade pattern (GP 3, GP 4 or GP 5) and adjacent normal glandular epithelium. No hormonal treatment or radiotherapy was administered to the patients before surgery. Patient profiles, including GS, are summarized in Tables 1 and 2.

\section{Laser Capture Microdissection (LCM) and miRNA Extraction}

Sections $8 \mu \mathrm{m}$ in thickness were placed on glass slides, deparaffinized, stained with hematoxylin, dehydrated, and placed on an ArcturusXT Nikon Eclipse Ti-E microdissection system for LCM according to the manufacturer's protocol. Tissue areas of interest were captured onto CapSure Macro LCM caps using infrared laser pulses. The LCM procedure was completed within 45 min per sample and total RNA (including miRNA) was extracted from microdissected FFPE tissues using an miRNeasy FFPE Kit (Qiagen, Hilden, Germany) according to the manufacturer's instructions. Briefly, FFPE tissue sections were lysed with proteinase $\mathrm{K}$ digestion after deparaffinating. Following heat treatment and centrifugation, the supernatant containing RNA was treated with DNase. After adding Buffer RBC and ethanol, the total miRNA was obtained by washing from the RNeasy MinElute column, and then stored at $-80^{\circ} \mathrm{C}$ until analysis. The RNA concentration was assessed using a NanoDrop 1000 spectrophotometer (Thermo Fisher Scientific, Waltham, Massachusetts, US). 


\section{Quantitative RT-PCR Analysis for miRNAs}

Extracted total RNA was reverse-transcribed into singlestranded cDNA using a Taqman MicroRNA Reverse Transcription Kit and real-time PCR was performed using first strand cDNA with TaqMan Fast Universal PCR Master Mix, according to the manufacturer's instructions (Thermo Fisher Scientific, Waltham, Massachusetts, US). Samples was normalized to U6 as an internal control, and Taqman miRNA assays used for the analysis were as follows: miR1-5p, miR-21-5p, miR-30d-5p, miR-100-5p, miR-145-5p, miR-224-5p, and miR-708-5p (Thermo Fisher Scientific, Waltham, Massachusetts, US). Briefly, cDNA was synthesized from $10 \mathrm{ng}$ of total RNA in a $5 \mu \mathrm{L}$ reaction volume using an miRNA-specific RT primer and a TaqMan miRNA Reverse Transcription Kit. All reactions were performed using an Eppendorf Mastercycler EP Gradient $\mathrm{S}$ instrument (Eppendorf, Germany) under the following conditions: $16^{\circ} \mathrm{C}$ for $30 \mathrm{~min}, 42^{\circ} \mathrm{C}$ for $30 \mathrm{~min}$, and at $85^{\circ} \mathrm{C}$ for $5 \mathrm{~min}$. Next, miRNA expression was evaluated using TaqMan miRNA Assays. Each $20 \mu \mathrm{L}$ PCR reaction contained $10 \mu \mathrm{L}$ of $2 \times$ Universal PCR Master Mix, $1.0 \mu \mathrm{L}$ of $20 \times$ TaqMan miRNA Assay Mix, and $1.0 \mu \mathrm{L}$ of RT product. Real-time PCR was performed under the following conditions: $95^{\circ} \mathrm{C}$ for $10 \mathrm{~min}$ for enzyme activation, followed by 40 cycles at $95^{\circ} \mathrm{C}$ for $15 \mathrm{sec}$ and $60^{\circ} \mathrm{C}$ for $1 \mathrm{~min}$. The average $\mathrm{Ct}$ values for the control ( $\mathrm{Ct}$ control) and the sample (Ct sample) were determined. These values were used to obtain the sample fold-change in gene expression relative to that of the control using the $2-\Delta \Delta \mathrm{Ct}$ method.

\section{Gene Ontology (GO) and Pathway Analysis}

Prediction of miRNA target genes was carried out using MiRWalk 3.0, miRDB and Targetscan7.2. The selected target genes were then deposited to the Metascape for GO annotation and Kyoto Encyclopedia of Genes and Genomes (KEGG) pathway enrichment analysis. $^{32}$ Besides, the other four datasets, including Reactome, KEGG disease, PANTHER pathway and NGHRI_GWAS_Catalog were also explored for enrichment analysis. Furthermore, the predicted targets of miRWalk 3.0 were submit to its own GO and pathway analysis to avoid the key information missing after only considering the intersection of predicted targets.
Table I Clinical Parameters of Study Cohort I and 2

\begin{tabular}{|c|c|c|}
\hline Study Population & RP Cohort & Biopsy Cohort \\
\hline No. patients & $n=53$ & $n=107$ \\
\hline Age (y) & $\begin{array}{c}65.8 \pm 7.6 \\
(66,6 I-72)\end{array}$ & $\begin{array}{c}68.6 \pm 8.2 \\
(69,63.5-74.5)\end{array}$ \\
\hline \multirow[t]{2}{*}{ PSA (ng/mL) } & $25.0 \pm 24.1$ & $23.0 \pm 29.1$ \\
\hline & $(16.0,10.1-29.4)$ & $(|2.5,8.9-2| . I)$ \\
\hline \multicolumn{2}{|c|}{ The highest GS at biopsy } & $3+3,32$ \\
\hline & & $\begin{array}{c}3+4,24 \\
3+5,3 \\
4+3,29 \\
4+4,13 \\
4+5,6\end{array}$ \\
\hline GS at RP & $\begin{array}{c}3+3,9 \\
3+4,10 \\
4+3,10 \\
4+4,8 \\
4+5,8 \\
5+4,8\end{array}$ & $\begin{array}{c}3+3,32 \\
3+4,35 \\
4+3,36 \\
4+5,4\end{array}$ \\
\hline
\end{tabular}

Note: Data are presented as mean \pm standard deviation (median, interquartile range).

Abbreviations: PSA, prostate specific antigen; RP, Radical prostatectomy; GS, Gleason score.

\section{Statistical Analysis}

The experiments were carried out in triplicate and the data presented were representative of three independent experiments. All results were presented as the mean with standard deviation (SD). Statistical analysis was conducted using the SPSS 22.0 software (SPSS Inc, Chicago, IL) and the GraphPad version 7.0 (GraphPad Software, La Jolla, CA). The average miRNA expression in normal tissue compared with that in malignant tissue was determined by Student's $t$-test and One-way ANOVA. Mann-Whitney test was used when the data does not follow a normal distribution. The diagnostic performance was evaluated using receiver operating characteristic (ROC) curves, and the area under the ROC curve (AUC) was calculated to evaluate the diagnostic value. The optimal cut-off value, sensitivity, and specificity were determined by calculating the Youden index. Using multiple logistic regression with the enter method, the clinical variables were entered and investigated as predictors of upgrading. The variables considered for entry into the model included age, the percentage of positive cores, PSA, prostate-specific antigen density (PSAD) and the relative 
Table 2 Characteristics of Biopsy GS6 Patients With or Without Gleason Upgrading Upon Subsequent RP in Cohort 3

\begin{tabular}{|c|c|c|c|c|}
\hline \multirow[t]{2}{*}{ No. patients } & Overall & Nonupgrading & Upgrading & \multirow[t]{2}{*}{$P$} \\
\hline & $n=119$ & $n=66,55.5 \%$ & $n=53,44.5 \%$ & \\
\hline Age (y) & $\begin{array}{c}69.0 \pm 6.5 \\
(69,65-73)\end{array}$ & $\begin{array}{c}68.1 \pm 6.7 \\
(68.5,64-71)\end{array}$ & $\begin{array}{c}70.1 \pm 6.1 \\
(70,66-75)\end{array}$ & 0.086 \\
\hline $\mathrm{PV}(\mathrm{mL})$ & $\begin{array}{c}48.8 \pm 31.6 \\
(37.5,29.1-62.4)\end{array}$ & $\begin{array}{c}45.5 \pm 22.1 \\
(37.1,29.1-63.3)\end{array}$ & $\begin{array}{c}51.5 \pm 37.8 \\
(37.5,29.1-57.9)\end{array}$ & 0.922 \\
\hline PSA (ng/mL) & $\begin{array}{c}14.7 \pm 13.8 \\
(12,7.3-17.1)\end{array}$ & $\begin{array}{c}15.0 \pm 16.5 \\
(10.4,7.4-14.3)\end{array}$ & $\begin{array}{c}14.2 \pm 9.6 \\
(12.8,7.3-18.7)\end{array}$ & 0.317 \\
\hline PSAD $\left(\mathrm{ng} / \mathrm{mL}^{2}\right)$ & $\begin{array}{c}0.39 \pm 0.57 \\
(0.24,0.15-0.43)\end{array}$ & $\begin{array}{c}0.40 \pm 0.75 \\
(0.22,0.13-0.40)\end{array}$ & $\begin{array}{c}0.38 \pm 0.37 \\
(0.25,0.17-0.46)\end{array}$ & 0.255 \\
\hline$\%$ of Positive cores & $\begin{array}{c}0.34 \pm 0.21 \\
(0.30,0.17-0.42)\end{array}$ & $\begin{array}{c}0.34 \pm 0.21 \\
(0.25,0.17-0.42)\end{array}$ & $\begin{array}{c}0.35 \pm 0.21 \\
(0.33,0.17-0.42)\end{array}$ & 0.631 \\
\hline miR-I45-5p & $\begin{array}{c}157.9 \pm 158.7 \\
(100.5,62.1-178.7)\end{array}$ & $\begin{array}{c}211.6 \pm 190.4 \\
(156.5,78.8-320.0)\end{array}$ & $\begin{array}{c}92.0 \pm 63.5 \\
(79.1,42.6-123.8)\end{array}$ & $<0.001$ \\
\hline
\end{tabular}

Note: Data are presented as mean \pm standard deviation (median, interquartile range).

Abbreviations: RP, radical prostatectomy; PV, prostate volume; PSA, prostate specific antigen; PSAD, PSA density.

expression level of miR-145-5p. $P<0.05$ was considered statistically significant.

\section{Results}

\section{Relative Expression of miRNAs Between the Gleason Patterns 3, 4 and 5}

To determine the expression of miRNAs associated with the $\mathrm{GP}$ of $\mathrm{PCa}$, we investigated seven miRNAs, including miR1-5p, miR-21-5p, miR-30d-5p, miR-100-5p, miR-145-5p, miR-224-5p, and miR-708-5p, all of which are implicated in $\mathrm{PCa}$ progression. Firstly, we explored the differential expression of seven miRNAs between GPs and adjacent normal tissues using RP samples (patient set 1). As shown in Figure 1, compared with normal samples, the relative expression of miR-224-5p in each GP was significantly decreased, the expression of miR-145-5p was downregulated in GP4 and GP5 but not in GP3, while the expression of miR-1-5p was downregulated in GP4 but not in GP3 or GP5. Then the miRNA expression among the different GPs was investigated. We found that the relative expression of miR$145-5 p$ in GP4 was significantly lower than that in GP3, and no other differential expression was found in this study.

\section{Altered miRNA Expression Among the Same GP with Different GS}

To explore whether miRNA expression in PCa with the same GP differed according to GS, we analyzed miRNA expression in GP4 and GP3 separately. In GP4, samples from GS7 or GS $\geq 8$ were evaluated, but no significant difference in miRNA expression associated with GS was detected (Supplementary Figure 1). However, in GP3, expression of miR-145-5p, miR-224-5p and miR-708-5p in samples obtained from GS6 was significantly higher compared with that of GS7 (Figure 2). Tissues from GP5 were excluded from the analysis, as all samples were obtained from GS9.

To verify the differential expression of miRNAs in the same GP3 between GS6 and GS7, a validation study using the biopsy samples was performed with patient set 2 . We found that the expression of miR-145-5p was significantly higher in GP3 tissues from the real GS6 compared with that associated with GP4, which was similar to the result obtained from the RP group. However, statisticallyinsignificant difference in the expression of miR-224-5p and miR-708-5p was found between GP3 samples from the real GS6 and that associated with GP4 (Figure 3).

\section{Association Between the Expression Level of miR-145-5p and Gleason Upgrading of Biopsy Samples Scored GS6}

To further investigate the performance of miR-145-5p in predicting the Gleason upgrading (GU) from GS6 biopsy samples, we detected its expression level using the cohort 3. The total GU was $44.5 \%(n=53)$ and the comparison of 
the nonupgrading, and upgrading groups was summarized in Table 2. As shown in Figure 4A, only the expression level of miR-145-5p decreased significantly in GU group. On ROC analysis, miR-145-5p yielded an AUC value of 0.705 (95\% CI, 0.612-0.798; $P<0.001$ ) with 50.8\% sensitivity and $84.8 \%$ specificity (Figure 4B). The AUC for other clinical parameters in predicting GU was 0.592 for age, 0.554 for PSA, 0.567 for PSAD and 0.525 for percent of positive cores respectively (Supplementary Figure 2). In multivariate analyses, miR-145-5p was an independent predictor of GU (Table 3).

\section{Target Gene Prediction of miR-I45-5p}

To further determine the biologic functions affected by miR-145-5p in PCa, the potential target genes were predicted by miRwalk 3.0 and two other miRNA prediction tools. The intersection of these three predicted target gene sets was integrated and visualized using Venn diagram (Supplementary Figure 3). Finally, 125 overlapping genes were consistently predicted by the three databases, suggesting that these genes might be involved in the miR-145$5 \mathrm{p}$-mediated functions and biological pathways in $\mathrm{PCa}$.

\section{GO Annotation and KEGG Enrichment Analyses}

To obtain insight into the biological roles of miR-145-5p in $\mathrm{PCa}, \mathrm{GO}$ annotation and KEGG pathway enrichment were analyzed through Metascape and KOBAS. ${ }^{32,33}$ As shown in Figure 5, these 125 target genes were mainly enriched in small GTPase mediated signal transduction and semaphoring-plexin signaling pathway with regard to biological processes (BPs) (Figure 5A). As for molecular functions (MFs), terms such as GTPase regulator activity and actin filament binding were enriched (Figure 5B). Regarding cellular components (CCs), the target genes were commonly enriched in the cell leading edge, postsynaptic specialization, contractile fiber and adherens junction (Figure 5C). Furthermore, the KEGG analysis revealed that adherens junction, endocytosis, dilated cardiomyopathy and HIF-1 signaling were significantly enriched pathways. Besides, the ecadherin stabilization pathway and smad2/3 nuclear pathway were enriched by Canonical Pathways enrichment, and the UV response DN and apical junction were enriched by HALLMARK enrichment (Figure 5D). In addition, databases such as KEGG DISEASE, and GWAS Catalog were also explored with KOBAS to further find out the relationship between miR-145-5p and diseases. The results showed that cardiovascular diseases and cancers were significantly enriched (Supplementary Figure 4), suggesting a potential correlation between miR-145-5p and PCa.

In view of missing key information from merely taking the intersection of the predicted targets into consideration, an enrichment analysis was also performed on all predicted target genes with the only miRWalk. ${ }^{34}$ Consequently, 6035 target genes with a miRWalk score $>1$ were used for the functional enrichment analysis. The results showed that cell junction assembly, neuron projection guidance and regulation of GTPase activity were enriched with regard to $\mathrm{BPs}$ (Supplementary Figure 5A).
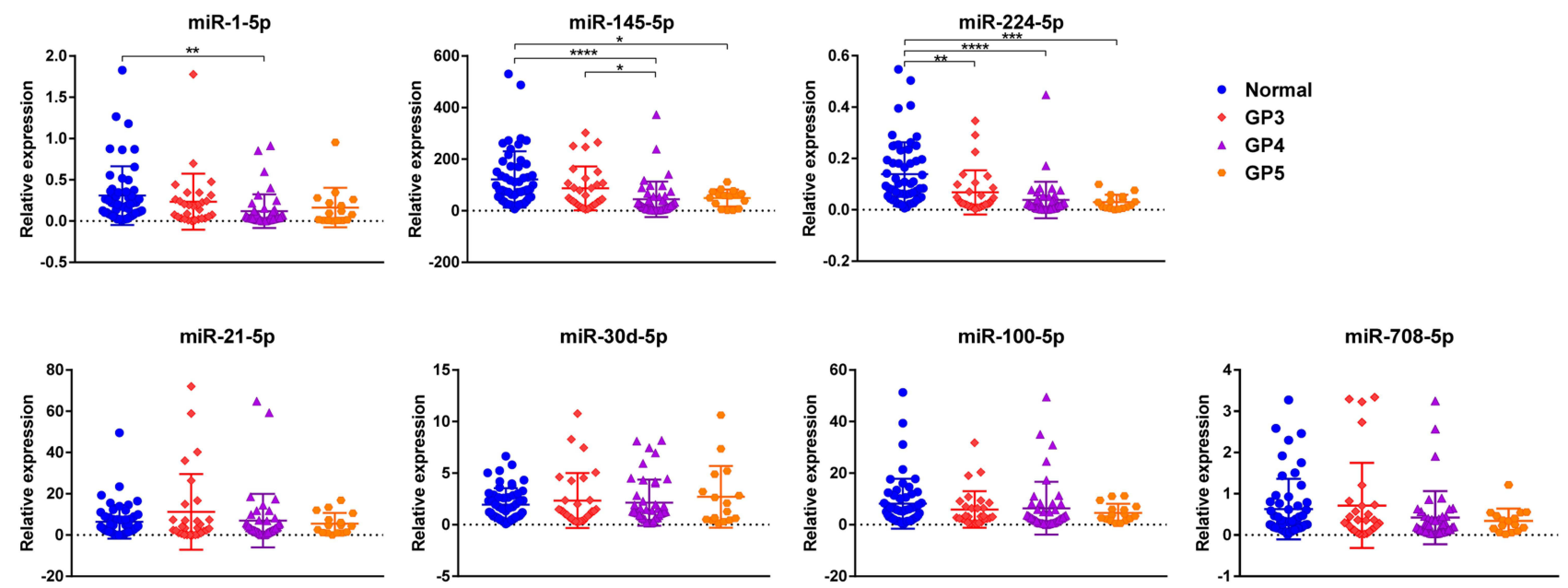

Figure I Relative expression of selected miRNAs between the Gleason Patterns 3, 4 and 5. Differential expression of selected miRNAs in tumor tissues of each GP (GP3: $n=29$, GP4: $n=44$, and GP5: $n=16$, respectively) compared to adjacent normal tissues $(n=53)$ as measured by RT-PCR. Only the statistically significant comparisons were noted by asterisks. $* \mathrm{P}<0.05$; $* \mathrm{P}<0.01$ I; $* * \mathrm{P}<0.001$; $* * * * \mathrm{P}<0.000 \mathrm{I}$.

Abbreviation: GP, Gleason Pattern. 

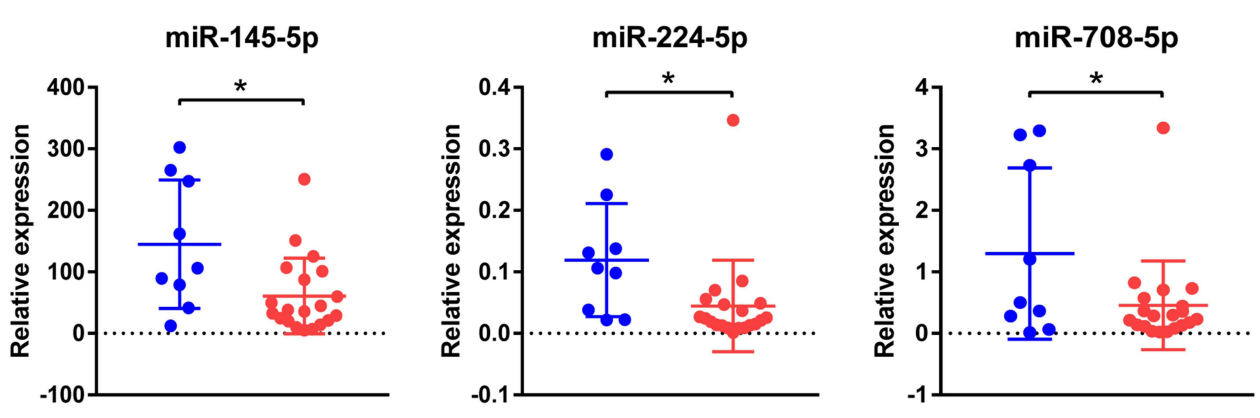

- GS6

- GS7
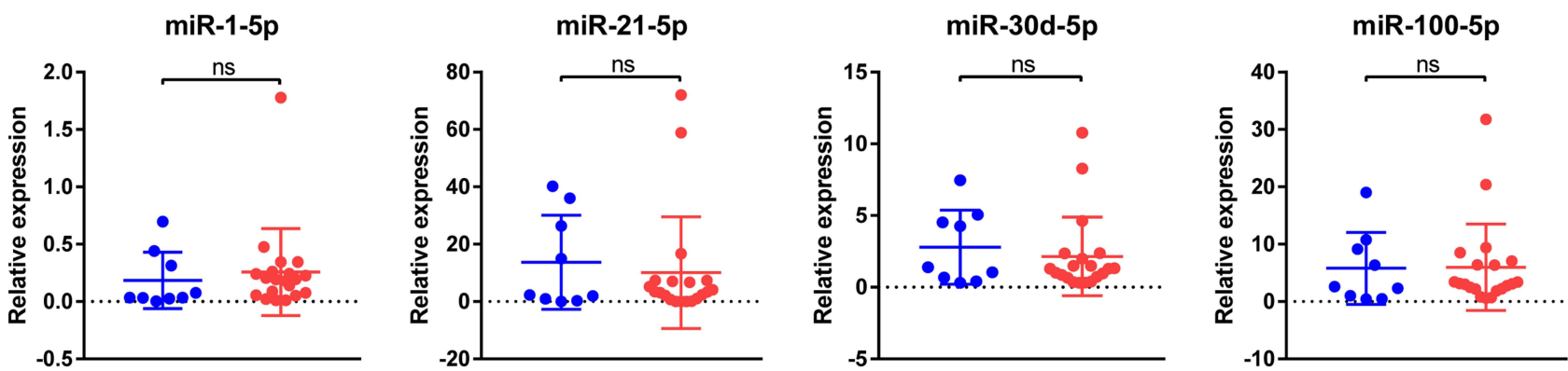

Figure 2 Relative expression of selected miRNAs in GP3 cancer tissues from pure GS 6 tumors and GS 7 tumors using cohort I. Differential expression of selected miRNAs in GP3 cancer tissues from pure GS 6 tumors $(n=9)$ and GS 7 tumors (either $4+3$ or $3+4, n=20$ ) using patient set $I$. * $P<0.05$.

Abbreviations: GS, Gleason score; ns, not significant.

As for MFs, symporter activity, solute:cation symporter activity, GTPase regulator activity and small GTPase binding were significantly enriched (Supplementary Figure 5B), indicating a close association between miR145-5p and the GTPase activity. Regarding CCs, the apical part of cell and synaptic membrane were enriched (Supplementary Figure 5C). In addition, terms such as pathway in focal adhesion were enriched In the KEGG pathway analysis (Supplementary Figure 5D), indicating that miR-145-5p might be involved in the structure remodeling in the carcinogenesis of PCa.

\section{Discussion}

Because of the prevalence of the PSA test and the modified Gleason grading system from the International Society of Urological Pathology, more cancers have been assigned a GS7 score or higher and there has been a more homogeneous assignment of a GS6 score. ${ }^{4,35}$ Patients with pure GP3 disease have an excellent prognosis. ${ }^{36-38}$ After a recent review of 14,000 RP specimens using the updated Gleason system, no lymph node metastases were identified in men with GS6 confirmed upon prostatectomy. ${ }^{39}$ The 10 year PCa-specific survival for pathological GS6 is $100 \%,{ }^{40}$
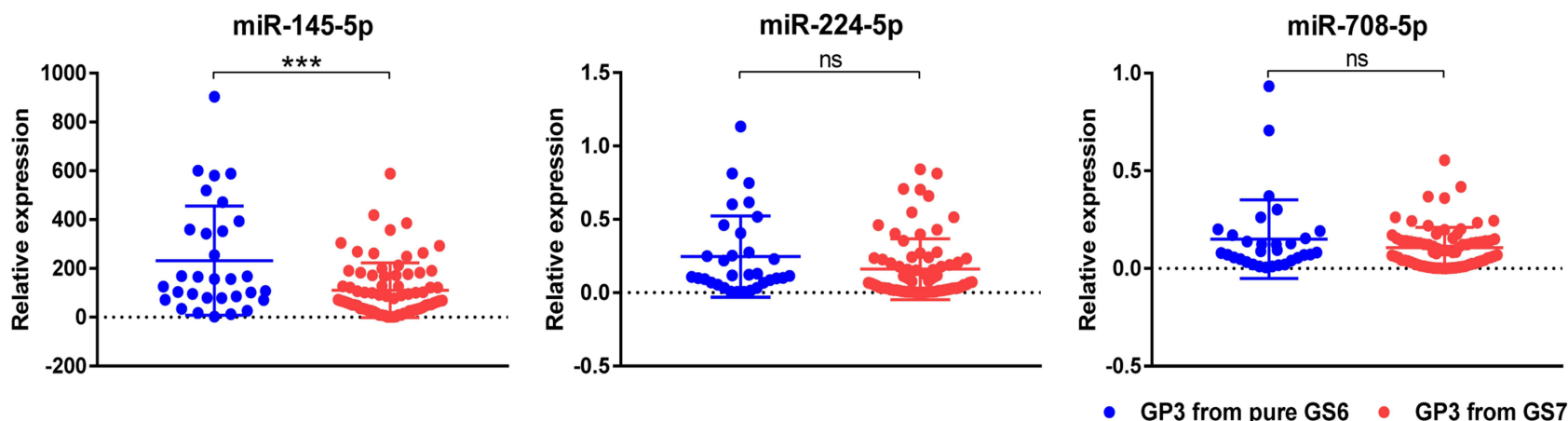

Figure 3 Validation of the expression of selected miRNAs in prostate cancer biopsy samples. Validation of the expression of selected miRNAs in prostate cancer biopsy samples. Scatter diagrams representing the tissue expression level of three miRNAs in GP3 from 107 FFPE PCa biopsy samples. We detected significant decrease in the expression of miR-145-5p in GP3 areas from pure GS6 tumors $(n=32)$ as compared to GP3 areas from GS7 (either 4+3 or 3+4, $n=75$ ). ***P<0.00।.

Abbreviation: ns, not significant. 
A

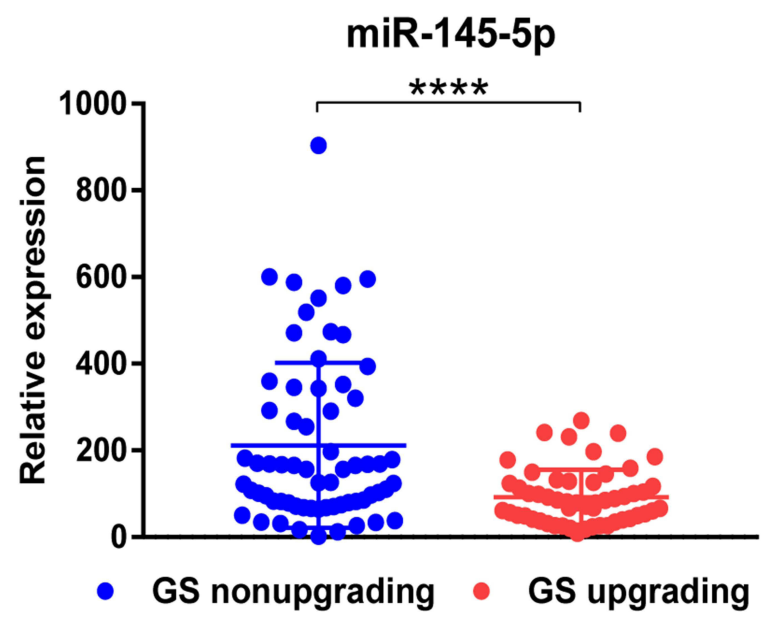

B

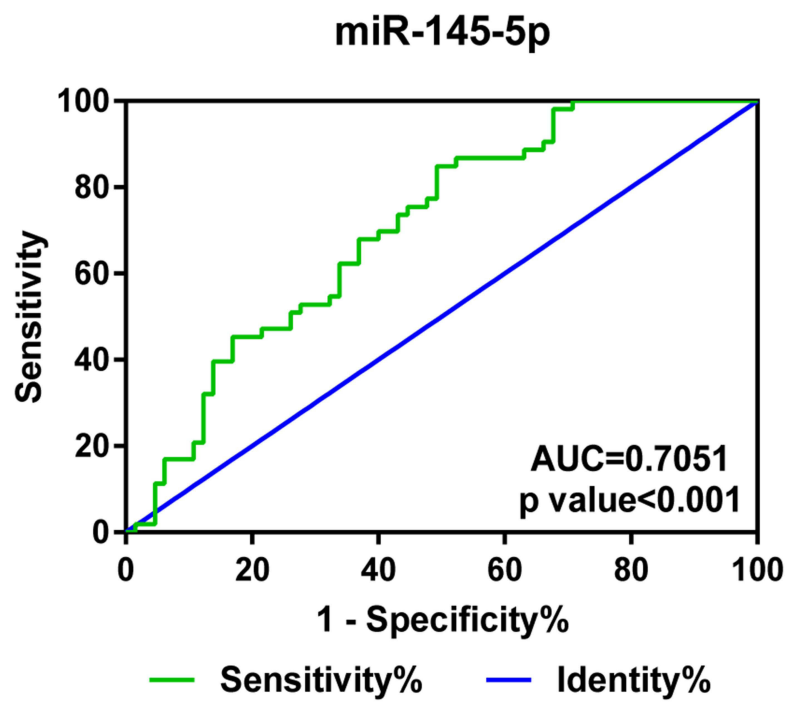

Figure 4 Association between the expression level of miR-I45-5p and Gleason upgrading (GU) of biopsy samples scored GS6. (A) Differential expression of miR-I45-5p in GS6 biopsy samples with GU in the following RP relative to those with GNU. (B) Receiver operating characteristic curve for miR-I45-5p. **** P<0.000I.

Abbreviation: AUC, area under the curve.

whereas the 15-year PCa-specific mortality rate is less than $1.2 \% .{ }^{41}$ If a given patient's prostate harbored only GS6 tumors, these patients could be safely monitored and select active surveillance (AS) as a surrogate for immediate intervention. ${ }^{42}$ Most cohorts use grade progression on serial biopsies to GS $3+4$ or higher as a threshold for intervention. Accordingly, cases of overtreatment could be avoided, as well as the inconvenience following radical intervention.

However, selection and monitoring of patients during AS can be inaccurate owing to the currently used PSA and repeat biopsy protocols. The GU was reported as $36 \%$ in patients whose biopsy pathologies were GS6. ${ }^{43}$ Recent studies have indicated that GU was associated with increased risk of biochemical recurrence (BR) and worse outcomes after RP. ${ }^{44-47}$ It is likely that sampling imprecision at the time of diagnosis is a major reason for GU upon

Table 3 Multivariate Analysis of Predictors for Gleason Upgrading in Cohort 3

\begin{tabular}{|l|c|c|}
\hline Variables & OR (Cl:95\%) & $P$ \\
\hline Age, y & $1.08 I(0.99 I-I .179)$ & 0.077 \\
PSA $(\mathrm{ng} / \mathrm{mL})$ & $1.058(0.956-1.17 I)$ & 0.279 \\
PSAD $\left(\mathrm{ng} / \mathrm{ml}^{2}\right)$ & $0.384(0.03 \mathrm{I}-4.734)$ & 0.455 \\
\% of positive cores & $1.090(0.098-12.17 \mathrm{I})$ & 0.944 \\
miR-I $45-5 \mathrm{P}$ & $0.990(0.985-0.996)$ & 0.001 \\
\hline
\end{tabular}

Abbreviations: $\mathrm{Cl}$, confidence interval; $\mathrm{OR}$, odds ratio. subsequent biopsies or RP. ${ }^{48-51}$ Thus, molecular and genetic researches are needed to facilitate the determination whether a patient harbors only GS6 cancer in the absence of RP and aid in the selection of patients for AS.

It is known that the most frequent somatic genomic aberrations in PCa include ETS family, TP53, and PTEN. ${ }^{52}$ Besides, germline or somatic aberrations in the DNA damage repair genes are found in $19 \%$ of primary $\mathrm{PCa}$ and almost $23 \%$ of metastatic castration-resistant $\mathrm{PCa}$ and compromise genomic integrity. ${ }^{52}$ However, the current nomograms have shown no additive value in predicting GU. ${ }^{48}$ It was reported that patients with pure GS6 tumors in subsequent RP samples had a lower possibility of PTEN loss in GP3 regions compared with that of patients with GS7 tumors. ${ }^{53}$ In addition, GP3 areas from GS7 tumors exhibited a greater rate of chromosome 8q24 gain and chromosome $8 \mathrm{p}$ loss. Meanwhile, Lotan et al revealed that pure GS6 tumor tissues at biopsy with lost PTEN detected by immunohistochemistry displayed an increased rate of GU at RP compared with that of tumors without PTEN loss. ${ }^{54}$ The findings based on the above literature suggest that GP3 lesions are molecularly distinct based on their presence in the context of GS6 or GS7 tumors.

Prior studies have provided evidence that miRNAs are closely associated with tumorigenesis and PCa progression. For example, angiogenesis plays a critical role in the development and spread of PCa. MiRNAs could regulate endothelial cells via non-cell-autonomous, as well as 
A

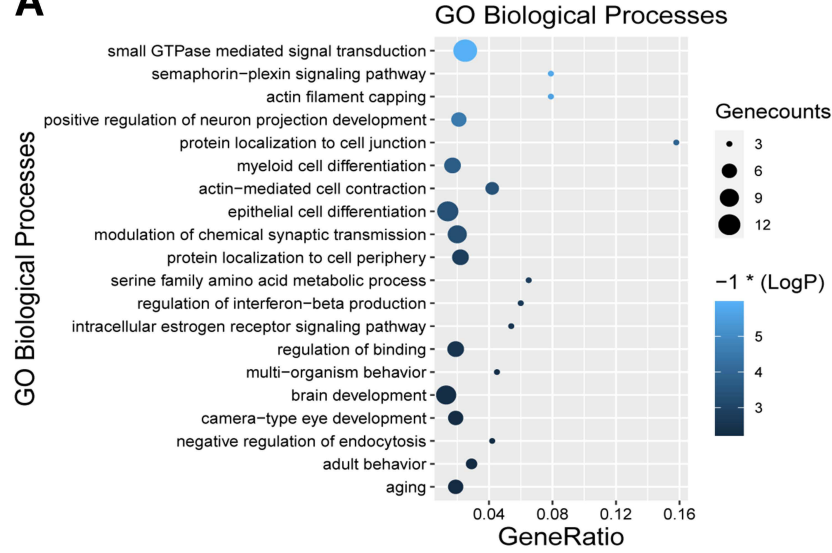

C

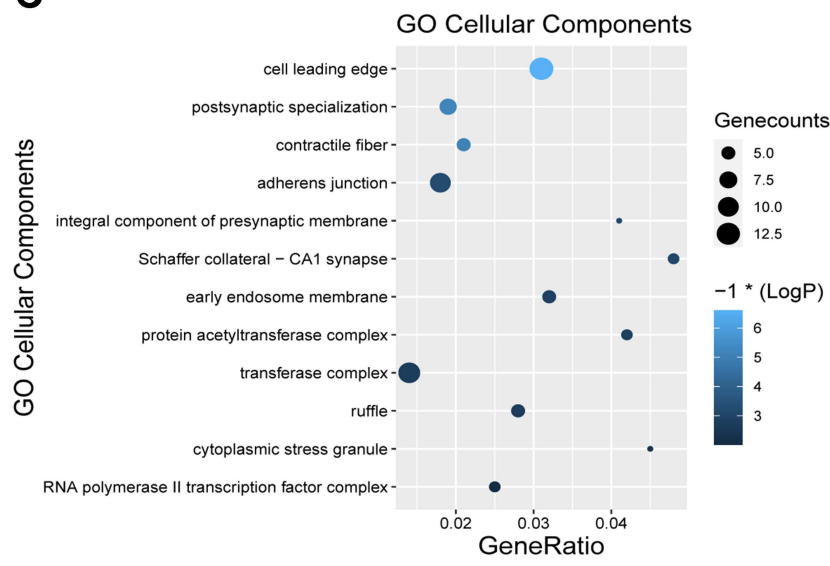

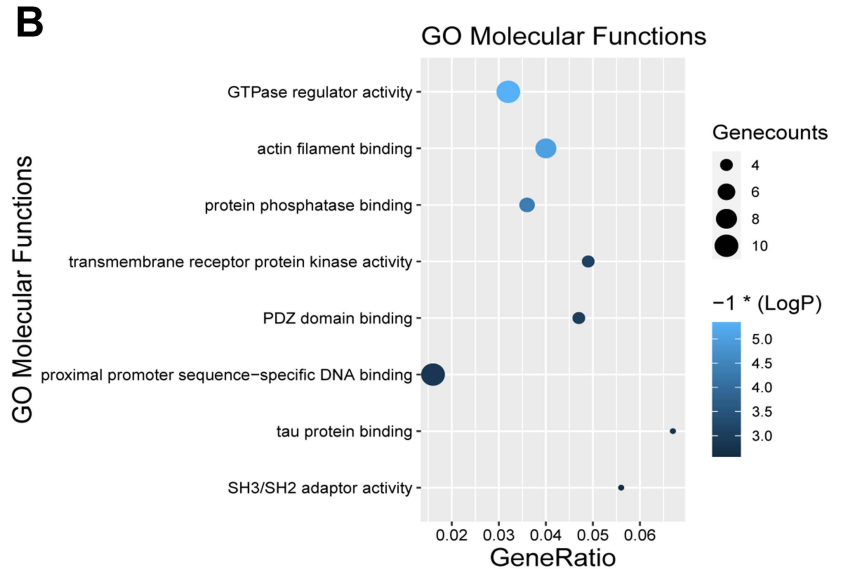

D

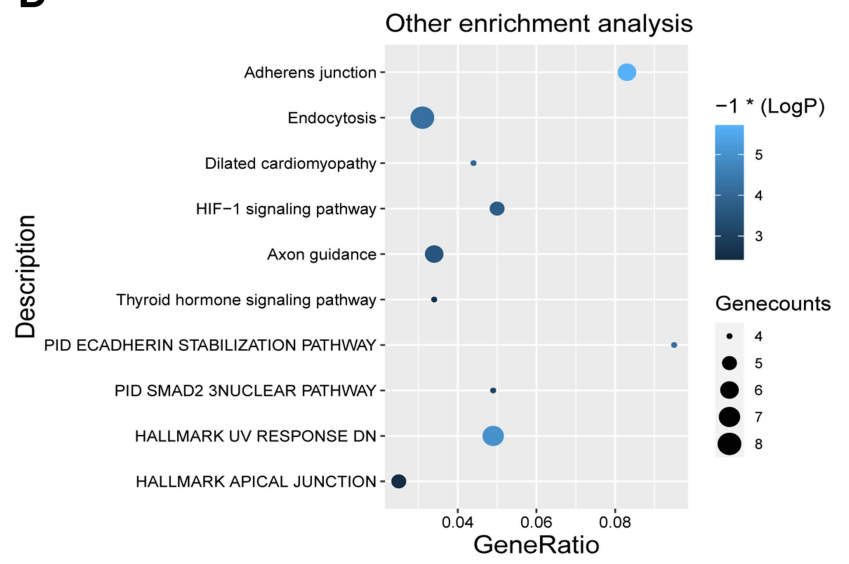

Figure 5 Gene ontology (GO) annotation and KEGG pathway enrichment analysis of predicted target genes of miR-145-5p. GO and KEGG analysis results. Terms with $\mathrm{P}<$ 0.01 were visualized as a bubble plot, as the more genes included, the bigger the size. Lighter colors indicate smaller P-values. (A) Enriched terms of GO biological process (BPs). (B) Enriched terms of GO molecular functions (MFs). (C) Enriched terms of GO cellular compounds (CCs). (D) Enriched terms of KEGG pathway, Canonical Pathways and Hallmark.

cell-autonomous techniques, and therefore control angiogenesis and PCa progression. ${ }^{55}$ Thus, single miRNAs or miRNA expression profiles could be potential biomarkers for PCa based on their stability and detectability in biopsy tissue and body fluids. ${ }^{56,57}$ In this study, we selected seven miRNAs that were involved in PCa. miR-1 was reported to be downregulated in primary $\mathrm{PCa}$ and further reduced in distant metastasis. ${ }^{18}$ Overexpression of miR-1 led to growth inhibition and down-regulation of genes associated with cell cycle progression, mitosis, DNA replication/ repair and actin dynamics via regulation of c-Met/AKT/ mTOR signaling pathway. ${ }^{17,18}$ miR-21 was overexpressed in patients with low-risk PCa, and could be an independent predictor of BR. ${ }^{19,20}$ Increased miR-21/AR expression mediate its tumor-promoting function by attenuating TGF $\beta$-mediated Smad2/3 activation, cell growth inhibition, cell migration and apoptosis. ${ }^{21}$ miR-30d overexpression was observed in both PCa cells and clinical specimens. Through MYPT1/c-JUN/VEGFA pathway,
miR-30d promoted angiogenesis and was associated with positive metastasis and BR, and reduced overall survival of PCa patients. ${ }^{22}$ Another study revealed that miR-30d, miR-181a and miR-199a-5p cooperatively suppressed the endoplasmic reticulum chaperone and signaling regulator GRP78 and GRP78-mediated chemoresistance. ${ }^{23}$ Except as an independent predictor of BR, lower expression of miR-100 in plasma was observed in high-grade compared to low-grade $\mathrm{PCa}$ at biopsy. ${ }^{24,25}$ miR-224 expression was significantly downregulated in PCa. Patients with PCa who were positive for miR-224 had significantly enhanced progression-free survival intervals, ${ }^{29}$ while miR-224 downregulation was significantly associated with poor BR-free survival. ${ }^{30}$ Mechanism research revealed that miR-224 inhibited proliferation, migration, and invasion of $\mathrm{PCa}$ cells by downregulating TRIB1 or direct/indirect regulation of pathways related to the cell cycle and cellular adhesion and motility. ${ }^{29,30}$ Besides, it was reported that high GABRE miR-452 miR-224 promoter methylation 
was significantly associated with $\mathrm{BR}^{28}$ Low miR-708 expression was associated significantly with poor survival outcome, tumor progression, and recurrence. ${ }^{31}$ Further research showed that reduced miR-708 expression led to PCa initiation, progression, and development by regulating the expression of CD44 as well as AKT2. ${ }^{31}$

Some miRNAs have been found to be associated with GS, however, most analyses have compared PCa samples with those of normal tissues. To date, only two studies have reported the relative expression of miRNAs among GPs. Tsuchiyama et al found that the expression of miR$182-5 p$ was increased in the GP4 from GS8 or GS9 cancer samples compared with that from GS7. ${ }^{58}$ Lichner et al compared global miRNA expression between pure areas of each Gleason pattern and identified miRNAs that were differentially expressed between the different patterns. ${ }^{8}$ The group identified extracellular matrix, focal adhesion kinase, and mitogen-activated protein kinase pathways as significant pathways targeted by these miRNAs. ${ }^{8}$ Overexpression of miR-29c, miR-34a and miR-141 could repress genes involved in ECM-mediated pathways, and decrease cell proliferation and migration. Besides, miR$29 \mathrm{c}$ and miR-34a could influence actin cytoskeleton organization and androgen receptor localization. ${ }^{8}$ However, no further research was conducted to investigate the relative expression of these miRNAs in samples with the same GP with different GS. These studies indicate that specific miRNAs could be evaluated to assist in determining whether patients with GS6 biopsies are at a higher risk of harboring a previously unsampled higher-grade prostate tumor.

Our study found that expression of miR-145-5p in GP3 was higher in the real GS6 compared with that in GS7 cancer, which was consistent with prior studies showing that miR-145-5p acted as tumor suppressor in $\mathrm{PCa}$ and was associated with high Gleason score, PSA and bone metastasis. ${ }^{26,27}$ Mechanism researches revealed that miR145 in PCa was associated with DNA methylation and p53 mutation and suppressed cell proliferation, migration and invasion through targeting fascin-1, SWAP70 and human enhancer of filamentation $1 .^{26,59}$ In a recent study, a significant difference for miR-145 was observed in the GU group by detecting from tumor tissues upon RP. ${ }^{20}$ While the predictive value of miR-145 for GU was not statistically significant in that research, our study discovered that miR-145-5p yielded an AUC value of 0.705 (95\% CI, 0.612-0.798; $P<0.001$ ) with $50.8 \%$ sensitivity and $84.8 \%$ specificity. Multivariate analyses showed that miR-145-5p was an independent predictor of GU. Furthermore, after identifying 125 potential target genes of miR-145-5p, we performed GO and KEGG pathway analyses to show the possible and crucial regulatory pattern of miR-145-5p. While small GTPase mediated signal transduction, GTPase regulator activity and cell leading edge were enriched in GO annotation, the adherens junction and endocytosis were the most significant pathways in KEGG pathway enrichment analysis. As a regulatory target by miR-145-5p, ${ }^{60}$ the small GTPase Rac1 initiates a series of signaling networks, including regulatory complex of actin cytoskeleton remodeling, activation of protein kinases (PAKs, MAPKs) and transcription factors (NFkB, Wnt//-catenin/TCF, STAT3, Snail), indicating a close association with the neoplastic progression from dysplasia to the metastatic cascade. ${ }^{61}$ Furthermore, recent research has reported that as a member of bromodomain and extraterminal domain protein family, BRD4 stabilization led to enhanced expression of the RAC1 and cholesterol-biosynthesis-associated genes together with activation of AKT-mTORC1 signaling in SPOP-mutant $\mathrm{PCa}$, further suggesting a close connection between GTPase and PCa. ${ }^{62}$

\section{Conclusions}

Our results show that early detection of miR-145-5p in prostate biopsy samples scored GS6 is useful in determining the aggressiveness of a given GS6 lesion and predicting GU upon subsequent biopsies or RP. It is hoped that in the future, miRNAs, along with molecular and genetic markers, can be applied in addition to GS to provide a more appropriate timing of active intervention for $\mathrm{PCa}$ patients with biopsy samples scored $3+3=6$.

\section{Abbreviations}

AUC, the area under the ROC curve; AS, active surveillance; BP, biological process; BR, biochemical recurrence; $\mathrm{CC}$, cellular component; FFPE, formalin-fixed, paraffinembedded; GO, Gene oncology; GP, Gleason pattern; GS, Gleason score; GU, Gleason upgrading; KEGG, Kyoto Encyclopedia of Genes and Genomes; LCM, Laser capture microdissection; MF, molecular function; miRNAs, MicroRNAs; $\mathrm{PCa}$, prostate cancer; PSA, prostate-specific antigen; PSAD, prostate-specific antigen density; ROC, receiver operator characteristics; RP, radical prostatectomy; SD, standard deviation. 


\section{Data Sharing Statement}

All data generated that are relevant to the results presented in this article are included in this article. Other data that were not relevant for the results presented here are available from the corresponding author Dr. Wang upon reasonable request.

\section{Ethics Approval and Consent to Participate}

Written informed consent was obtained from each patient included and the protocol of all study cohorts were approved by the Clinical Research Ethics Committee of Shanghai general hospital and the Ethics Committee of Fudan University Shanghai Cancer Center. All methods were performed in accordance with the Declaration of Helsinki.

\section{Acknowledgments}

We thank Dr. Peipei Zhang (Department of Pathology, Ruijin Hospital, Shanghai Jiao Tong University School of Medicine, Shanghai, China) for their excellent pathological technology help.

\section{Author Contributions}

All authors made a significant contribution to the work reported, whether that is in the conception, study design, execution, acquisition of data, analysis and interpretation, or in all these areas; took part in drafting, revising or critically reviewing the article; gave final approval of the version to be published; have agreed on the journal to which the article has been submitted; and agree to be accountable for all aspects of the work.

\section{Funding}

This study was funded by grants from National Natural Science Foundation of China (81570607), Three-year action plan for promoting clinical skills and clinical innovation in municipal hospitals of Shanghai Shenkang (16CR2003A), National Natural Science Foundation for Young Scholars of China (81902566), Shanghai Jiaotong University Medical-Engineering Cross Research Fund (YG2019QNA53), Zhejiang Medical and health science and technology plan project (2019KY713). All these study sponsors have no roles in the study design, in the collection, analysis and interpretation of data.

\section{Disclosure}

The authors have declared that no conflict of interest exists.

\section{References}

1. Siegel RL, Miller KD, Jemal A. Cancer statistics, 2019. CA Cancer J Clin. 2019;69(1):7-34. doi:10.3322/caac.21551

2. Chen W, Zheng R, Baade PD, et al. Cancer statistics in China, 2015. CA Cancer J Clin. 2016;66(2):115-132. doi:10.3322/caac.21338

3. Epstein JI, Egevad L, Amin MB, Delahunt B, Srigley JR, Humphrey PA. The 2014 International Society of Urological Pathology (ISUP) consensus conference on gleason grading of prostatic carcinoma: definition of grading patterns and proposal for a new grading system. Am J Surg Pathol. 2016;40(2):244-252. doi: $10.1097 /$ pas. 0000000000000530

4. Epstein JI, Zelefsky MJ, Sjoberg DD, et al. A contemporary prostate cancer grading system: a validated alternative to the gleason score. Eur Urol. 2016;69(3):428-435. doi:10.1016/j.eururo.2015.06.046

5. Epstein JI, Feng Z, Trock BJ, Pierorazio PM. Upgrading and downgrading of prostate cancer from biopsy to radical prostatectomy: incidence and predictive factors using the modified Gleason grading system and factoring in tertiary grades. Eur Urol. 2012;61 (5):1019-1024. doi:10.1016/j.eururo.2012.01.050

6. Bartel DP. MicroRNAs: genomics, biogenesis, mechanism, and function. Cell. 2004;116(2):281-297. doi:10.1016/s0092-8674(04) 00045-5

7. Schickel R, Boyerinas B, Park SM, Peter ME. MicroRNAs: key players in the immune system, differentiation, tumorigenesis and cell death. Oncogene. 2008;27(45):5959-5974. doi:10.1038/ onc. 2008.274

8. Lichner Z, Ding Q, Samaan S, et al. miRNAs dysregulated in association with Gleason grade regulate extracellular matrix, cytoskeleton and androgen receptor pathways. J Pathol. 2015;237(2):226-237. doi: $10.1002 /$ path. 4568

9. Fang YX, Gao WQ. Roles of microRNAs during prostatic tumorigenesis and tumor progression. Oncogene. 2014;33(2):135-147. doi:10.1038/onc.2013.54

10. Aghdam SG, Ebrazeh M, Hemmatzadeh M, et al. The role of microRNAs in prostate cancer migration, invasion, and metastasis. J Cell Physiol. 2019;234(7):9927-9942. doi:10.1002/jcp.27948

11. Rizzo M. Mechanisms of docetaxel resistance in prostate cancer: the key role played by miRNAs. Biochim Biophys Acta Rev Cancer. 2021;1875(1):188481. doi:10.1016/j.bbcan.2020.188481

12. Saxby H, Mikropoulos C, Boussios S. An update on the prognostic and predictive serum biomarkers in metastatic prostate cancer. Diagnostics. 2020;10(8). doi:10.3390/diagnostics 10080549

13. Ghafouri-Fard S, Shoorei H, Taheri M. Role of microRNAs in the development, prognosis and therapeutic response of patients with prostate cancer. Gene. 2020;759:144995. doi:10.1016/j. gene.2020.144995

14. Fabris L, Ceder Y, Chinnaiyan AM, et al. The potential of MicroRNAs as prostate cancer biomarkers. Eur Urol. 2016;70 (2):312-322. doi:10.1016/j.eururo.2015.12.054

15. Martínez-González LJ, Sánchez-Conde V, González-Cabezuelo JM, et al. Identification of MicroRNAs as viable aggressiveness biomarkers for prostate cancer. Biomedicines. 2021;9(6):646. doi:10.3390/ biomedicines 9060646

16. Valera VA, Parra-Medina R, Walter BA, Pinto P, Merino MJ. microRNA expression profiling in young prostate cancer patients. $J$ Cancer. 2020;11(14):4106-4114. doi:10.7150/jca.37842

17. Gao S, Zhao Z, Wu R, Wu L, Tian X, Zhang Z. MiR-1 inhibits prostate cancer PC3 cells proliferation through the Akt/mTOR signaling pathway by binding to c-Met. Biomed Pharmacother. 2019;109:1406-1410. doi:10.1016/j.biopha.2018.10.098 
18. Hudson RS, Yi M, Esposito D, et al. MicroRNA-1 is a candidate tumor suppressor and prognostic marker in human prostate cancer Nucleic Acids Res. 2012;40(8):3689-3703. doi:10.1093/nar/gkr1222

19. Li T, Li RS, Li YH, et al. miR-21 as an independent biochemical recurrence predictor and potential therapeutic target for prostate cancer. $J$ Urol. 2012;187(4):1466-1472. doi:10.1016/j. juro.2011.11.082

20. Kurul NO, Ates F, Yilmaz I, Narli G, Yesildal C, Senkul T. The association of let-7c, miR-21, miR-145, miR-182, and miR-221 with clinicopathologic parameters of prostate cancer in patients diagnosed with low-risk disease. Prostate. 2019;79(10):1125-1132. doi:10.1002/pros. 23825

21. Mishra S, Deng JJ, Gowda PS, et al. Androgen receptor and microRNA-21 axis downregulates transforming growth factor beta receptor II (TGFBR2) expression in prostate cancer. Oncogene. 2014;33(31):4097-4106. doi:10.1038/onc.2013.374

22. Lin ZY, Chen G, Zhang YQ, et al. MicroRNA-30d promotes angiogenesis and tumor growth via MYPT1/c-JUN/VEGFA pathway and predicts aggressive outcome in prostate cancer. Mol Cancer. 2017;16 (1):48. doi:10.1186/s12943-017-0615-x

23. Su SF, Chang YW, Andreu-Vieyra C, et al. miR-30d, miR-181a and miR-199a-5p cooperatively suppress the endoplasmic reticulum chaperone and signaling regulator GRP78 in cancer. Oncogene. 2013;32 (39):4694-4701. doi:10.1038/onc.2012.483

24. Leite KR, Tomiyama A, Reis ST, et al. MicroRNA-100 expression is independently related to biochemical recurrence of prostate cancer J Urol. 2011;185(3):1118-1122. doi:10.1016/j.juro.2010.10.035

25. McDonald AC, Vira M, Walter V, et al. Circulating microRNAs in plasma among men with low-grade and high-grade prostate cancer at prostate biopsy. Prostate. 2019;79(9):961-968. doi:10.1002/ pros. 23803

26. Xu WX, Liu Z, Deng F, et al. MiR-145: a potential biomarker of cancer migration and invasion. Am J Transl Res. 2019;11 (11):6739-6753.

27. Avgeris M, Stravodimos K, Fragoulis EG, Scorilas A. The loss of the tumour-suppressor miR-145 results in the shorter disease-free survival of prostate cancer patients. $B r \quad J$ Cancer. 2013;108 (12):2573-2581. doi:10.1038/bjc. 2013.250

28. Kristensen H, Haldrup C, Strand S, et al. Hypermethylation of the GABRE $\sim \operatorname{miR}-452 \sim$ miR-224 promoter in prostate cancer predicts biochemical recurrence after radical prostatectomy. Clin Cancer Res. 2014;20(8):2169-2181. doi:10.1158/1078-0432.Ccr-13-2642

29. Mavridis K, Stravodimos K, Scorilas A. Downregulation and prognostic performance of microRNA 224 expression in prostate cancer. Clin Chem. 2013;59(1):261-269. doi:10.1373/clinchem.2012.191502

30. Lin ZY, Huang YQ, Zhang YQ, et al. MicroRNA-224 inhibits progression of human prostate cancer by downregulating TRIB1. Int J Cancer. 2014;135(3):541-550. doi:10.1002/ijc.28707

31. Saini S, Majid S, Shahryari V, et al. miRNA-708 control of CD44(+) prostate cancer-initiating cells. Cancer Res. 2012;72(14):3618-3630. doi:10.1158/0008-5472.Can-12-0540

32. Zhou Y, Zhou B, Pache L, et al. Metascape provides a biologist-oriented resource for the analysis of systems-level datasets. Nat Commun. 2019;10(1):1523. doi:10.1038/s41467-01909234-6

33. Ai C, Kong L. CGPS: a machine learning-based approach integrating multiple gene set analysis tools for better prioritization of biologically relevant pathways. J Genet Genomics. 2018;45(9):489-504. doi:10.1016/j.jgg.2018.08.002

34. Sticht C, De La Torre C, Parveen A, Gretz N. miRWalk: an online resource for prediction of microRNA binding sites. PLoS One. 2018;13(10):e0206239. doi:10.1371/journal.pone.0206239

35. Berg KD, Thomsen FB, Nerstrøm C, et al. The impact of the 2005 International Society of Urological Pathology consensus guidelines on Gleason grading - A matched-pair analysis. BJU Int. 2016;117 (6):883-889. doi:10.1111/bju.13439
36. Pierorazio PM, Walsh PC, Partin AW, Epstein JI. Prognostic Gleason grade grouping: data based on the modified Gleason scoring system. BJU Int. 2013;111(5):753-760. doi:10.1111/j.1464410X.2012.11611.x

37. Kweldam CF, Wildhagen MF, Bangma $\mathrm{CH}$, van Leenders GJ. Disease-specific death and metastasis do not occur in patients with Gleason score $\leq 6$ at radical prostatectomy. BJU Int. 2015;116 (2):230-235. doi:10.1111/bju.12879

38. Wilt TJ, Brawer MK, Jones KM, et al. Radical prostatectomy versus observation for localized prostate cancer. $N$ Engl J Med. 2012;367 (3):203-213. doi:10.1056/NEJMoa1113162

39. Ross HM, Kryvenko ON, Cowan JE, Simko JP, Wheeler TM, Epstein JI. Do adenocarcinomas of the prostate with Gleason score (GS) $\leq 6$ have the potential to metastasize to lymph nodes? Am J Surg Pathol. 2012;36(9):1346-1352. doi:10.1097/ PAS.0b013e3182556dcd

40. Bul M, van den Bergh RC, Zhu X, et al. Outcomes of initially expectantly managed patients with low or intermediate risk screen-detected localized prostate cancer. BJU Int. 2012;110 (11):1672-1677. doi:10.1111/j.1464-410X.2012.11434.X

41. Eggener SE, Scardino PT, Walsh PC, et al. Predicting 15-year prostate cancer specific mortality after radical prostatectomy. J Urol. 2011;185(3):869-875. doi:10.1016/j.juro.2010.10.057

42. Klotz L, Vesprini D, Sethukavalan P, et al. Long-term follow-up of a large active surveillance cohort of patients with prostate cancer. $J$ Clin Oncol. 2015;33(3):272-277. doi:10.1200/jco.2014.55.1192

43. De Nunzio C, Pastore AL, Lombardo R, et al. The new Epstein gleason score classification significantly reduces upgrading in prostate cancer patients. Eur J Surg Oncol. 2018;44(6):835-839. doi:10.1016/j.ejso.2017.12.003

44. Corcoran NM, Hong MK, Casey RG, et al. Upgrade in Gleason score between prostate biopsies and pathology following radical prostatectomy significantly impacts upon the risk of biochemical recurrence. BJU Int. 2011;108(8 Pt 2):E202-E210. doi:10.1111/j.1464410X.2011.10119.x

45. Freedland SJ, Kane CJ, Amling CL, Aronson WJ, Terris MK, Presti JC. Upgrading and downgrading of prostate needle biopsy specimens: risk factors and clinical implications. Urology. 2007;69 (3):495-499. doi:10.1016/j.urology.2006.10.036

46. Blute ML, Shiau JM, Truong M, et al. A biopsy-integrated algorithm for determining Gleason 6 upgrading risk stratifies risk of active surveillance failure in prostate cancer. World J Urol. 2017;35 (5):729-735. doi:10.1007/s00345-016-1933-0

47. Boorjian SA, Karnes RJ, Crispen PL, et al. The impact of discordance between biopsy and pathological Gleason scores on survival after radical prostatectomy. J Urol. 2009;181(1):95-104; discussion 104. doi:10.1016/j.juro.2008.09.016

48. Wang SY, Cowan JE, Cary KC, Chan JM, Carroll PR, Cooperberg MR. Limited ability of existing nomograms to predict outcomes in men undergoing active surveillance for prostate cancer. BJU Int. 2014;114(6b):E18-e24. doi:10.1111/bju.12554

49. Tosoian JJ, Trock BJ, Landis P, et al. Active surveillance program for prostate cancer: an update of the Johns Hopkins experience. J Clin Oncol. 2011;29(16):2185-2190. doi:10.1200/jco.2010.32.8112

50. Sheridan TB, Carter HB, Wang W, Landis PB, Epstein JI. Change in prostate cancer grade over time in men followed expectantly for stage T1c disease. J Urol. 2008;179(3):901-4; discussion 904-5. doi:10.1016/j.juro.2007.10.062

51. Penney KL, Stampfer MJ, Jahn JL, et al. Gleason grade progression is uncommon. Cancer Res. 2013;73(16):5163-5168. doi:10.1158/ 0008-5472.Can-13-0427

52. Boussios S, Rassy E, Shah S, Ioannidou E, Sheriff M, Pavlidis N. Aberrations of DNA repair pathways in prostate cancer: a cornerstone of precision oncology. Expert Opin Ther Targets. 2021;25(5):329-333. doi:10.1080/14728222.2021.1951226 
53. Trock BJ, Fedor H, Gurel B, et al. PTEN loss and chromosome 8 alterations in Gleason grade 3 prostate cancer cores predicts the presence of un-sampled grade 4 tumor: implications for active surveillance. Mod Pathol. 2016;29(7):764-771. doi:10.1038/ modpathol.2016.63

54. Lotan TL, Carvalho FL, Peskoe SB, et al. PTEN loss is associated with upgrading of prostate cancer from biopsy to radical prostatectomy. Mod Pathol. 2015;28(1):128-137. doi:10.1038/ modpathol.2014.85

55. Ioannidou E, Moschetta M, Shah S, et al. Angiogenesis and anti-angiogenic treatment in prostate cancer: mechanisms of action and molecular targets. Int J Mol Sci. 2021;22(18):9926. doi:10.3390/ ijms22189926

56. Chiam K, Ricciardelli C, Bianco-Miotto T. Epigenetic biomarkers in prostate cancer: current and future uses. Cancer Lett. 2014;342 (2):248-256. doi:10.1016/j.canlet.2012.02.011

57. Ottman R, Nguyen C, Lorch R, Chakrabarti R. MicroRNA expressions associated with progression of prostate cancer cells to antiandrogen therapy resistance. Mol Cancer. 2014;13:1-21. doi:10.1186/ 1476-4598-13-1
58. Tsuchiyama $\mathrm{K}$, Ito $\mathrm{H}$, Taga $\mathrm{M}$, et al. Expression of microRNAs associated with Gleason grading system in prostate cancer: miR-182-5p is a useful marker for high grade prostate cancer. Prostate. 2013;73(8):827-834. doi:10.1002/pros.22626

59. Suh SO, Chen Y, Zaman MS, et al. MicroRNA-145 is regulated by DNA methylation and p53 gene mutation in prostate cancer. Carcinogenesis. 2011;32(5):772-778. doi:10.1093/carcin/bgr036

60. Lin KH, Kumar VB, Shanmugam T, et al. miR-145-5p targets paxillin to attenuate angiotensin II-induced pathological cardiac hypertrophy via downregulation of Rac 1, pJNK, p-c-Jun, NFATc3, ANP and by Sirt-1 upregulation. Mol Cell Biochem. 2021;476 (9):3253-3260. doi:10.1007/s11010-021-04100-w

61. Kotelevets L, Chastre E. Rac1 signaling: from intestinal homeostasis to colorectal cancer metastasis. Cancers. 2020;12(3):665. doi: $10.3390 /$ cancers 12030665

62. Zhang P, Wang D, Zhao Y, et al. Intrinsic BET inhibitor resistance in SPOP-mutated prostate cancer is mediated by BET protein stabilization and AKT-mTORC1 activation. Nat Med. 2017;23(9):1055-1062. doi: $10.1038 / \mathrm{nm} .4379$

\section{Publish your work in this journal}

Cancer Management and Research is an international, peer-reviewed open access journal focusing on cancer research and the optimal use of preventative and integrated treatment interventions to achieve improved outcomes, enhanced survival and quality of life for the cancer patient.
The manuscript management system is completely online and includes a very quick and fair peer-review system, which is all easy to use. Visit http://www.dovepress.com/testimonials.php to read real quotes from published authors. 